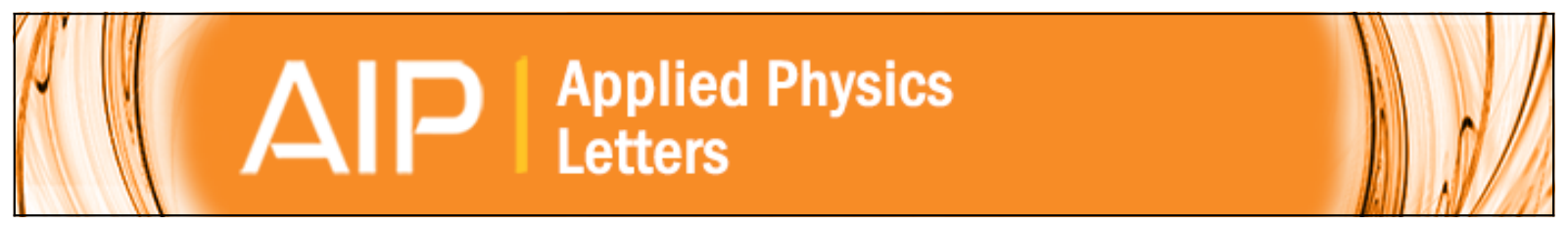

Strain relaxation and stress-driven interdiffusion in In As In Ga As In $\mathbf{P}$ nanowires

L. Nieto, J. R. R. Bortoleto, M. A. Cotta, R. Magalhães-Paniago, and H. R. Gutiérrez

Citation: Applied Physics Letters 91, 063122 (2007); doi: 10.1063/1.2764446

View online: http://dx.doi.org/10.1063/1.2764446

View Table of Contents: http://scitation.aip.org/content/aip/journal/apl/91/6?ver=pdfcov

Published by the AIP Publishing 


\title{
Strain relaxation and stress-driven interdiffusion in InAs/InGaAs/InP nanowires
}

\author{
L. Nieto, J. R. R. Bortoleto, ${ }^{\text {a) }}$ and M. A. Cotta ${ }^{\text {b) }}$ \\ Instituto de Física Gleb Wataghin, UNICAMP, CP 6165, 13083-970 Campinas, São Paulo, Brazil \\ R. Magalhães-Paniago \\ Departamento de Física, UFMG, CP 702, 30123-970 Belo Horizonte, Minas Gerais, Brazil \\ H. R. Gutiérrez \\ Department of Physics, Pennsylvania State University, University Park, Pennsylvania 16802-6300
}

(Received 23 May 2007; accepted 3 July 2007; published online 9 August 2007)

\begin{abstract}
The authors have investigated strain relaxation in InAs/InGaAs/InP nanowires (NW's). Transmission electron microscopy images show an additional stress field attributed to compositional modulation in the ternary layer, which disrupts NW formation and drives Ga interdiffusion into InAs, according to grazing incidence $\mathrm{x}$-Ray diffraction under anomalous scattering conditions. The strain profile along the NW, however, is not significantly affected when interdiffusion is considered. Results show that the InAs NW energetic stability is preserved with the introduction of ternary buffer layer in the structure. (C) 2007 American Institute of Physics. [DOI: 10.1063/1.2764446]
\end{abstract}

Considerable amount of work has been carried out on self-assembled quantum dots and wires due to the attractive physical properties expected from these nanostructures. ${ }^{1}$ In particular, InP-based InAs nanostructures are also interesting for their ability to work as light emitters in the $1.30-1.60 \mu \mathrm{m}$ long-wavelength region. ${ }^{2-7}$ In contrast to the InAs/GaAs system, the formation process of InAs nanostructures on $\mathrm{InP}$ substrates is characterized by the lower strain $(\sim 3.2 \%)$ between InAs and the buffer layers, and complicated by possible chemical alloying with the substrate ${ }^{2,3}$ anisotropic stress relaxation, ${ }^{7}$ and the large number of possible materials for the buffer layer. ${ }^{2-7}$ Experimental results suggest that stress relaxation ${ }^{7}$ and/or the chemical composition $^{4}$ of the buffer layer (InP, InAlAs, or InGaAs) have key roles in configuring the final shape of the InAs nanostructures. However, the mechanism associated with the formation of a specific surface morphology of nanostructures still remains under discussion. ${ }^{8}$

In this letter, we analyze the role of InGaAs/InP buffer layers grown under different conditions on InAs nanowires (NW's) shape and size. Our results suggest that InGaAs compositional modulation disrupts the formation of InAs NW's due to the associated periodic stress field. When the modulation is not present, strain relaxation in InAs/InGaAs NW's is very similar to the InAs/InP case, suggesting a minor role for using a different buffer layer.

The samples were grown on vicinal $\left(2^{\circ}\right.$ off towards $\langle 111\rangle$ A) (001)InP substrates by chemical beam epitaxy. A $120 \mathrm{~nm}$ thick InP buffer layer was deposited followed by the nominally lattice-matched InGaAs film, with thickness varying between 10 and $100 \mathrm{~nm}$ and a planar surface for the subsequent InAs growth at $490{ }^{\circ} \mathrm{C}$ and $2.4 \AA / \mathrm{s}$. The main differences regarding sample structure are due to $\mathrm{InGaAs}$ thickness $(12 \mathrm{~nm}$ for sample B and $100 \mathrm{~nm}$ for samples C and D) and $\mathrm{As}_{2}$ overpressure during InGaAs growth (a three-

\footnotetext{
${ }^{\text {a) }}$ Present address: Campus Experimental de Sorocaba, GPM, UNESP, Sorocaba, 18087-180 São Paulo, Brazil.

b) Electronic mail: monica@ifi.unicamp.br
}

fold overpressure was used for sample D as compared to B and C). Sample A is a reference sample where InAs was deposited directly on InP. All samples were cooled down in vacuum in order to preserve the actual nanostructure shape ${ }^{9}$ and analyzed by in-air atomic force microscopy (AFM) and cross sectional transmission electron microscopy (TEM) using a JEM 3010 URP $300 \mathrm{kV}$. Nanowire strain relaxation was investigated by the grazing incidence $\mathrm{x}$-ray diffraction (GIXD).${ }^{10,11}$ We have used GIXD radial and angular scans under anomalous scattering conditions ${ }^{12}$ in order to evaluate the presence of $\mathrm{Ga}$ within the InAs nanostructures. The difference in intensity between radial scans for the (2-20) reflection taken at $10 \mathrm{keV}\left(I_{2}\right)$ and at the $K$-edge absorption energy for $\mathrm{Ga}, E=10.367 \mathrm{keV}\left(I_{1}\right)$ provided the Ga concentration in the nanostructure, ${ }^{12}$ according to

$$
C^{\mathrm{Ga}}=\frac{1}{2}\left(f^{\mathrm{In}}+f^{\mathrm{As}}\right) \frac{1-\sqrt{I_{1} / I_{2}}}{\sqrt{I_{1} / I_{2}}\left(f_{2}^{\mathrm{Ga}}-f^{\mathrm{In}}\right)-\left(f_{1}^{\mathrm{Ga}}-f^{\mathrm{In}}\right)} .
$$

where the In and As scattering factors $f$ are constant at the energies used, $C^{\mathrm{In}}+C^{\mathrm{Ga}}=0.5$ and $C^{\mathrm{As}}=0.5$. Subsequently, angular scans at the Ga $K$-edge energy as well as TEM and AFM images ${ }^{11-13}$ allowed us to create a profile of the actual Ga concentration along the height of the InAs NW.

Figure 1 shows AFM topographies of the InAs NW's. We can observe that InAs/InP wires [Fig. 1(a), sample A] run quite uniformly along the [1-10] direction with base width $\sim 32 \mathrm{~nm}$. However, for sample B, where InAs was grown on an $\sim 12 \mathrm{~nm}$ thick InGaAs film [Fig. 1(b)], the NW's are usually shorter, with a few dots showing up on the surface. In this case, the NW base width is $\sim 45 \mathrm{~nm}$. The shape of the NW's is altered even further when the thickness of the InGaAs film is increased to $100 \mathrm{~nm}$ (sample C), as shown in Fig. 1(c). In this case, the NW shape anisotropy is also altered, and tilted structures-as compared to the previous images-show up on the surface. However, Fig. 1(d) shows that it is possible to regain InAs/InGaAs shapes similar to InAs/InP (sample D). In this case, the InGaAs thickness is also $\sim 100 \mathrm{~nm}$, and the only difference in growth conditions is the larger $\mathrm{As}_{2}$ overpressure. The InAs NW's are 


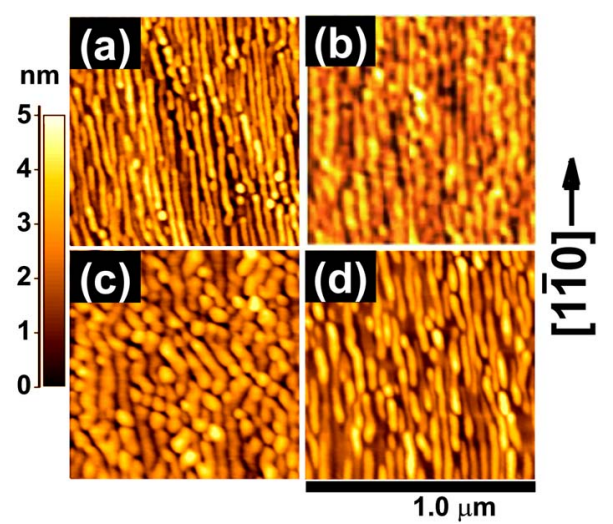

FIG. 1. (Color online) AFM images of InAs NW's. (a) InAs/InP (sample A) and InAs/InGaAs/InP with InGaAs thickness (b) $12 \mathrm{~nm}$ (sample B), (c) $100 \mathrm{~nm}$ (sample C), and (d) $100 \mathrm{~nm}$, grown under larger $\mathrm{As}_{2}$ overpressure (sample D). The heights and NW's densities depend on growth condition and thickness of the InGaAs buffer layers. However, samples A and D (B and C) show similar height distributions.

more regular and uniform, similar to those grown on InP, and the base width dropped to $\sim 35 \mathrm{~nm}$. The density and height distribution of the NW's also varies depending on InGaAs growth conditions.

The formation of InAs NW's on InP has been attributed to InP surface anisotropy ${ }^{14}$ and to anisotropic stress relaxation linked to the P-As interface. ${ }^{7}$ However, InAs NW's on InGaAs/InP have already been reported. ${ }^{5}$ In our case, we observe that the shape and direction of the NW's on the surface depends on the buffer layer thickness, suggesting the role of a volumetric effect. This effect could be attributed to different elastic properties of InGaAs and InP films. Thin InGaAs films would not hinder completely the presence of the InP substrate underneath. However, elastic parameters for these materials are very similar and could not explain the observed behavior. Moreover, this effect would not be suppressed by changing InGaAs growth conditions, as shown in Fig. 1(d).

Figure 2 shows $g=(-2-20)$ dark field TEM images of samples B, C, and D. An intensity contrast in the InGaAs layer can be seen in Figs. 2(a) and 2(b); this contrast is periodically more uniform and clear along the layer for the thicker InGaAs film. These results can be associated with the compositional modulation within the InGaAs film, most likely due to the low $\mathrm{As}_{2}$ overpressure used. ${ }^{15-17} \mathrm{~A}$ twodimensional periodic pattern along the [100] and [010] directions for In and $\mathrm{Ga}$ rich regions has been reported for compositionally modulated III-V layers. ${ }^{16-18}$ If this is the case for our InGaAs films, the modulation strain field will be rotated $45^{\circ}$ from the [1-10] direction of the InAs/InP NW's long axis. ${ }^{14}$ From TEM, we estimate the period of the compositional modulation inside the InGaAs layer of samples B and $\mathrm{C}$ in the range of $45-50 \mathrm{~nm}$. These values are similar to the
NW base width for these samples. Moreover, we can observe in Fig. 1(c) that NW's are more discontinuous and often oriented at about $45^{\circ}$ out of [1-10] direction. Larger group $\mathrm{V}$ overpressures usually hinder the formation of compositional modulation in ternary layers. ${ }^{16}$ Indeed, the TEM image of sample D [Fig. 2(c)] shows only the contrast usually associated with the strain field of the InAs nanostructures.

The periodic strain field associated with the modulation may disrupt the formation of InAs NW's since it represents an extra driving force for In diffusion along the surface. As a result, InAs accumulates on top of In-rich regions on the InGaAs surface, especially for the first few layers, which will eventually determine the onset of three-dimensional islanding. For thin InGaAs films, where the stress field is weaker, the main result is the altered aspect ratio of InAs NW's, with a larger base width. For a thicker InGaAs buffer layer, however, both shape and direction of the nanostructures on the surface are affected.

Figure 3(a) shows the anisotropic strain relaxation for NW's grown on InGaAs and InP. We have plotted the strain $\varepsilon_{h k l}=\left(d_{\mathrm{InAs}}-d_{h k l}\right) / d_{\mathrm{InAs}}$ as a function of the height of the NW along both the [220] and [2-20] directions. The height values in the figure were calculated considering the NW base width (obtained by GIXD and TEM) and $\{114\}$ sidewall facets, ${ }^{11}$ still preserved when InAs is deposited on InGaAs. The curves for samples A and D are very similar. The InAs NW grown on InGaAs (sample D) relaxes slightly faster than for InAs/InP (sample A). Anomalous scans taken at the Ga $K$-edge energy show no sign of Ga interdiffusion from InGaAs into the InAs NW's for sample D [Fig. 3(b)]. These results suggest that the InAs strain relaxation should not strongly depend on the buffer material.

A completely different result, however, is obtained for sample B, where the shape of the NW's is still preserved in spite of the compositional modulation observed in the InGaAs buffer layer. In this case, GIXD data taken at $10 \mathrm{keV}$ [Fig. 3(a)] indicate that strain relaxation-especially for the [220] direction-is quite different from the previous case. However, anomalous radial scans, shown in the inset of Fig. 3(b), indicate a non-negligible Ga concentration within the InAs NW's for this sample. The Ga profile obtained using angular scans is shown in Fig. 3(b). This result suggests that interdiffusion takes place due to the extra strain field provided by compositional modulation. Once a nanometer-sized, two-dimensional periodic strain field is built in the buffer layer, strain-driven kinetic processes can take place during InAs deposition. The relatively fast growth rates for InAs used here should not account for Ga surface diffusion as the origin of the observed behavior. Keeping in mind that the NW long axis should be rotated with regard to the modulation pattern, ${ }^{17}$ we can assume that sections of the InAs NW's are deposited on Ga-rich regions of the buffer layer, thus providing the driving force for $\mathrm{Ga}$ interdiffusion.
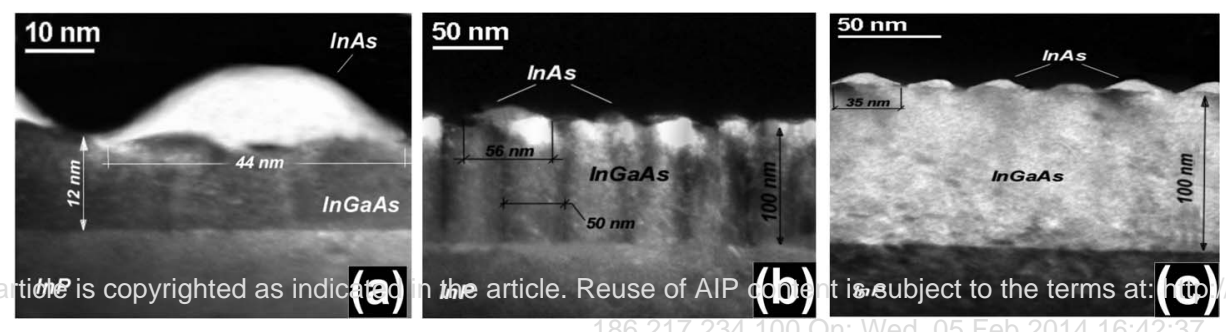

FIG. 2. High resolution TEM [dark field for $g=(-2-20)]$ cross section images of InAs/InGaAs NW's. Bright and dark regions in InGaAs show typical mass contrast associated with compositional modulation in samples (a) B, (b) C, and (c) D. 

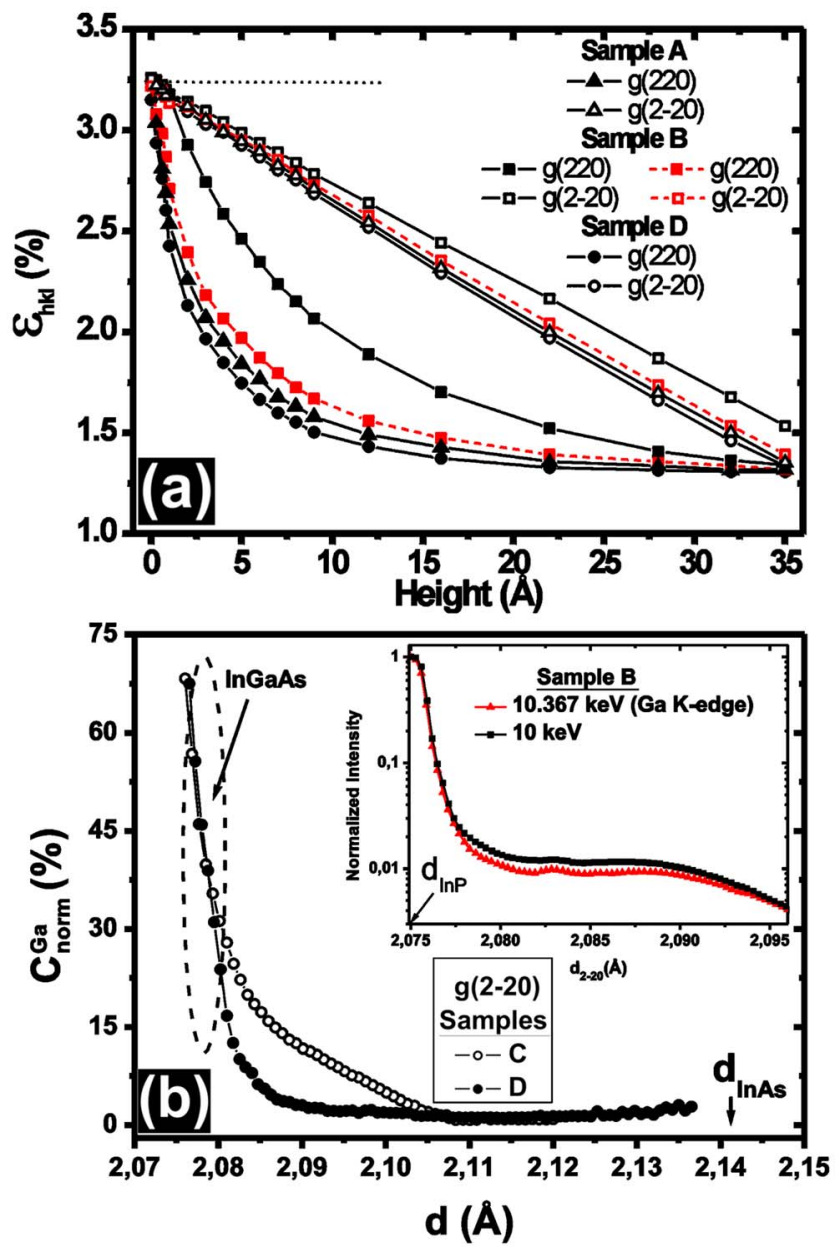

FIG. 3. (Color online) Strain and Ga concentration profiles obtained from GIXD for samples A, B, and D. (a) Strain relaxation along the height of the NW for the [220] and [2-20] directions. (b) Ga concentration profile for the [2-20] direction for samples $\mathrm{B}$ and $\mathrm{D}$, normalized to the maximum $\mathrm{Ga}$ concentration from the InGaAs buffer layer (considered as 100\%). The curves for $g=(220)$ are very similar. The inset shows the radial scans for InAs taken at both energies. The Ga concentration for sample B, shown in (b), was considered for strain calculations as well. The dashed curve for sample B in (a) was obtained assuming a Vegard law correction.

The Ga profiles shown in Fig. 3(b) reflect an average of the NW strain field probed by GIXD measurements. If we then recalculate the strain curve along the height of the nanostructure, assuming a Vegard law for the $\operatorname{In}(\mathrm{Ga})$ As lattice parameter at each isostrain plane, we obtain the dashed curve, shown in Fig. 3(a), where $\varepsilon_{h k l}=\left(d_{\mathrm{In}_{x} \mathrm{Ga}_{1-x} \mathrm{As}}-d_{h k l}\right) /$ $d_{\mathrm{In}_{x} \mathrm{Ga}_{1-x} \mathrm{As}}$. We can notice that the actual strain for sample B is very similar to those from samples A and D, small differences likely related to the NW shape variations. Also, in the case of sample B, local Ga variations must exist within the NW's, which cannot be probed by GIXD.

In summary, the formation of InAs NW's on InGaAs/InP, under certain growth conditions, depends on the thickness of the InGaAs buffer layer. Our results suggest that compositional modulation in the buffer layer disrupts nanowire formation and may lead to Ga interdiffusion. The strain relaxation profile along the nanostructure, however, is very similar to the InAs/InP case if the modulation is not present in the buffer layer or the actual Ga concentration is considered. Our results show that the introduction of a ternary compound in between InAs/InP does not significantly affect InAs NW stability if proper parameters are chosen for the growth. This nanostructure stability is a suitable characteristic for band gap engineering and device applications.

One of the authors (L.N.) acknowledges scholarship from CNPq. This work was supported by FAPESP and CNPq. HRTEM and GIXD measurements were performed at LME and XRD-2 station, respectively, at the National Laboratory of Synchrotron Light (LNLS, Brazil).

${ }^{1}$ A. D. Yoffe, Adv. Phys. 50, 1 (2001).

${ }^{2}$ J. Brault, M. Gendry, G. Grenet, G. Hollinger, Y. Dieres, and T. Benyattou, Appl. Phys. Lett. 73, 2932 (1998).

${ }^{3}$ H. Yoon, Y. Moon, T. Lee, E. Yoon, and Y. Kim, Appl. Phys. Lett. 74, 2029 (1999).

${ }^{4}$ V. M. Ustinov, E. R. Weber, S. Ruvimov, Z. Liliental-Weber, A. E. Zhukov, A. Yu. Egorov, A. R. Kovsh, A. F. Tsatsulnikov, and P. S. Kopev, Appl. Phys. Lett. 72, 362 (1998).

${ }^{5}$ H. Li, T. Daniels-Race, and M. A. Hasan, Appl. Phys. Lett. 80, 1367 (2002).

${ }^{6}$ F. Suárez, D. Fuster, L. González, Y. González, J. M. García, and M. L. Dotor, Appl. Phys. Lett. 89, 091123 (2006).

${ }^{7}$ J. M. García, L. González, M. U. González, J. P. Silveira, Y. González, and F. Briones, J. Cryst. Growth 227, 975 (2001).

${ }^{8}$ G. Saint-Girons, A. Michon, I. Sagnes, G. Beaudoin, and G. Patriarche, Phys. Rev. B 74, 245305 (2006).

${ }^{9}$ H. R. Gutiérrez, M. A. Cotta, and J. R. R. Bortoleto, J. Appl. Phys. 92, 7523 (2002).

${ }^{10}$ I. Kegel, T. H. Metzger, A. Lorke, J. Peisl, J. Stangl, G. Bauer, J. M. Garcia, and P. M. Petroff, Phys. Rev. Lett. 85, 1694 (2000).

${ }^{11}$ H. R. Gutiérrez, R. Magalhães-Paniago, J. R. R. Bortoleto, and M. A. Cotta, Appl. Phys. Lett. 85, 3581 (2004).

${ }^{12}$ M. Sztucki, T. U. Schülli, T. H. Metzger, E. Beham, D. Schuh, and V. Chamard, Superlattices Microstruct. 36, 11 (2004).

${ }^{13}$ A. Malachias, R. Magalhães-Paniago, S. Kycia, and D. Gahill, J. Appl. Phys. 96, 3234 (2004).

${ }^{14}$ H. R. Gutiérrez, M. A. Cotta, and M. G. De Carvalho, Appl. Phys. Lett. 79, 3854 (2001).

${ }^{15}$ F. Peiró, J. C. Ferrer, A. Cornet, M. Calamiotou, and A. Georgakilas, Phys. Status Solidi A 195, 32 (2003).

${ }^{16}$ J. R. R. Bortoleto, H. R. Gutiérrez, M. A. Cotta, and J. Bettini, J. Appl. Phys. 101, 064907 (2007).

${ }^{17}$ J. R. R. Bortoleto, H. R. Gutiérrez, M. A. Cotta, and J. Bettini, Appl. Phys. Lett. 87, 013105 (2005).

${ }^{18}$ P. Henoc, A. Izrael, M. Quillec, and H. Launois, Appl. Phys. Lett. 40, 963 (1982). 